Canadian University Music Review

Canadian University Music Review

Revue de musique des universités canadiennes

\title{
Art mélodique et techniques de composition chez Massenet : exemple de la fonction diachronique du paradigme
}

\section{Gottfried R. Marschall}

\section{Numéro 6, 1985}

URI : https://id.erudit.org/iderudit/1014033ar

DOI : https://doi.org/10.7202/1014033ar

Aller au sommaire du numéro

Éditeur(s)

Canadian University Music Society / Société de musique des universités canadiennes

ISSN

0710-0353 (imprimé)

2291-2436 (numérique)

Découvrir la revue

Citer cet article

Marschall, G. R. (1985). Art mélodique et techniques de composition chez Massenet : exemple de la fonction diachronique du paradigme. Canadian University Music Review / Revue de musique des universités canadiennes, (6), 58-89. https://doi.org/10.7202/1014033ar

(c) Canadian University Music Society / Société de musique des universités canadiennes, 1985
Ce document est protégé par la loi sur le droit d'auteur. L'utilisation des services d'Érudit (y compris la reproduction) est assujettie à sa politique d'utilisation que vous pouvez consulter en ligne. 


\title{
ART MÉLODIQUE ET TECHNIQUES DE COMPOSITION CHEZ MASSENET: EXEMPLE DE LA FONCTION DIACHRONIQUE DU PARADIGME
}

\author{
Gottfried R. Marschall
}

0. Un compositeur dit de second rang comme l'est Jules Massenet, que peut-il nous apprendre sur l'évolution des styles et techniques de l'art, quelles réponses peut-il offrir à la discussion méthodologique?

0.1. Pour expliquer la singularité des oeuvres des grands génies, il faut regarder le travail des maîtres d'importance secondaire ${ }^{1}$ - encore que ce "déclassement " paraisse souvent injuste. Car ceux-ci représentent la norme stylistique de leur époque et font par-là apparaître la continuité et l'évolution des styles et structures. Tout porte à penser qu'il n'y a d'innovation que graduelle, relative : tendances latentes qui se font jour grâce à un " coup de pouce » décisif ; trouvailles apparentes préparées par mille détails guère visibles ; le choc des grands événements cachant les filiations réelles; le courage d'abandonner les poncifs et les développements qui ont dépassé leur paroxysme ... et pour sortir de l'impasse, pourquoi ne pas tenter de prendre les données à l'envers?

0.2. La synchronie pure semble en fait impossible. ${ }^{2}$ L'instantané ne montre qu'une réalité artificiellement arrêtée, une réalité en mouvement permanent où chaque détail a son histoire laquelle, par contre-coup, explique la présence de ce détail. Comment rendre compte du rôle de on, sans savoir qu'il remonte à homo ? Pourquoi 70 se dit soixante-dix en France, mais septante en Belgique et en Suisse ? La dodécaphonie n'apparaît-elle pas au moment précis où l'ambiguïté du chromatisme et de l'enharmonie a mené l'harmonie fonctionnelle à l'absurde ? Lors d'une analyse synchronique d'une langue $\mathrm{L}$, on constate que, au moment $\mathrm{t}_{\mathrm{A}}$ 
focalisé par l'analyse, chaque paradigme, dans chaque classe de composants de L, est arrivé à un stade précis d'une évolution, stade qui peut être celui d'une transition. ${ }^{3}$ Mais s'il y a parfois analogie entre paradigmes ou entre types d'évolution, les différents paradigmes ne se présentent pas, au moment ${ }^{\mathrm{A}} \mathrm{A}$, comme arrivés à des points analogues dans leur évolution respective. ${ }^{4}$ C'est pour cela que l'analyse purement synchronique se heurte parfois à des incompatibilités et que bon nombre de choses restent inexplicables.

Il s'agit donc de pourvoir chaque paradigme d'un indicateur de fonction diachronique. Bien que ce soit là un objectif idéal dont la réalisation posera des problèmes de détail, cette opération devrait s'appliquer à d'autres domaines qu'au seul langage, au moins à toute oeuvre humaine, y compris à la musique.

1. L'oeuvre musicale de Jules Massenet se prête particulièrement bien à ce genre d'analyse.

1.1. Les jugements prononcés sur l'importance de Massenet dans l'histoire de la musique français sont des plus divers et s'étendent des railleries d'un Gauthier-Villars ${ }^{5}$ jusqu'aux éloges d'un Louis Schneider $(1908 ; 1926)$ ou, de nos jours, à la discrète admiration d'un James Harding (1970). Pour les uns, Massenet était avant tout un imitateur dont l'oeuvre présente le panorama des styles en vogue à l'époque et les réminiscences de bon nombre de clichés d'autrefois, le tout étant saupoudré de sentimentalité. Pour les autres, il se rangeait en première ligne d'un courant de compositeurs désignés, non sans chauvinisme, comme "école française ". Les deux positions, si extrêmes soient-elles, ont à la fois tort et raison. Elles sont fausses parce qu'elles partent de critères secondaires, généraux, superficiels et en tout cas mal définis. Rares, pour ne pas dire inexistants, sont les auteurs qui prennent la peine d'analyser sérieusement la structure, le tissu, les composants, la genèse de l'oeuvre massenétiste.

1.2. Mais les opinions citées peuvent nous être utiles parce qu'elles font entrevoir deux choses qui semblent essentielles chez Massenet et justifient à elles seules l'analyse approfondie, que l'on prenne ou non Massenet pour un compositeur " de valeur ". D'une part, on repère dans la musique massenétiste un certain nombre de constantes ou stéréotypes allant de cellules rythmico-mélodiques jusqu'aux principes de construction et de pensée ; ces constantes permettent en grande partie de définir son langage personnel. D'autre part, il s'avère que ce langage personnel ne contient guère d'éléments radicalement nouveaux mais, tout en se nourrissant de 
sourses diverses, apporte très discrètement des orientations innovatrices. Massenet se situe au croisement de plusieurs traditions ou modes (grand opéra, style de Gounod, principes wagnériens, échos du Moyen-Âge, résonances religieuses, couleur locale et orientalisme), pour préparer la voie à l'impressionnisme (Debussy) et influencer le post-vérisme italien (Puccini). Son rôle est donc celui d'un médiateur qui aide à mieux comprendre la musique d'un Debussy, d'un Puccini et même certains éléments de la musique français du $\mathrm{XX}^{\mathrm{e}}$ siècle. Quant à Debussy, on a trop souvent souligné la nouveauté sans précédent de sa musique, ou bien, si précédent il y a, les chefs d'inspiration extra-européens, a-traditionnels, voire nettement insaisissables.

1.3. Massenet était avant tout compositeur de théâtre. Les oeuvres orchestrales ne peuvent que confirmer cette vocation par leur caractère pittoresque, illustratif, et par leur forme cyclique et rhapsodique. Le développement symphonique au sens stricte beethovenien - n'était visiblement pas sa préoccupation principale. Les mélodies - ou plutôt les romances - abondent, mais leur trait léger, leurs sujets anodins, leur manque de substance (musicale et littéraire) si souvent affirmés font que la plupart d'elles sombrent dans des tiroirs que même les descendants du compositeur s'interdisent d'ouvrir. Celles qui nous sont accessibles $^{6}$ révèlent ou confirment toutefois des traits typiques : absence de pesanteur, une pensée essentiellement mélodique, un sens aigu des propriétés du matériel langagier à transposer en musique.

2. Nous voici en face du premier souci de Massenet : homme de théâtre doté d'une plume lyrique et empreint de l'importance du verbe, le compositeur savait non seulement choisir ses livrets en fonction du potentiel musical et de la force évocatrice et émotionnelle contenus dans le sujet et dans les paroles mêmes, mais aussi rendre musicalement, avec une perfection étonnante, la prosodie de la langue française, avec toutes ses finesses et tous ses défis.

2.1 Cet effort s'inscrit dans une esthétique française qui se cristallise dans la seconde moitié du $\mathrm{XIX}^{\mathrm{e}}$ siècle ${ }^{7}$ mais qui avait toujours existé sans être réalisée avec succès dans tous les cas. La plupart du temps, elle traduisait la volonté de se démarquer des influences étrangères, notamment de l'opéra italien, volonté exprimée à plusieurs reprises, et paradoxalement, par des étrangers. Si Lully et Gluck entendaient purifier l'opéra en l'identifiant à un nouveau dramma per musica, ils ont par le même 
coup revalorisé le texte et réintroduit la simplicité et la correction de la déclamation. Grétry, dont la pratique tient à peine les promesses de la théorie, avait bien décelé, dans ses Mémoires (1789), les péchés d'une prosodie musicale maladroite. Le problème de l'opéra italien au Paris du $\mathrm{XIX}^{\mathrm{e}}$ siècle était aussi un problème d'intelligibilité des paroles. Réussir à l'Opéra de Paris, capital cosmopolite de l'Europe culturelle, signifiait, même pour les Italiens - voir Rossini, Donizetti, Verdi - de devoir composer sur des textes français, comme l'avait fait l'Allemand Meyerbeer. Le courant plus ou moins inauguré par Gounod, cette fameuse école française dont la cohérence reste à prouver, avait écrit sur sa bannière la promotion de la latinité musicale pour se démarquer, cette fois, de l'influence wagnérienne dont elle adopte et adapte malgré tout certains principes (abandon du morcellement formel, correspondance étroite de texte et musique). Faut-il auusi rappeler le fait que la chanson française moderne, d'un Brassens par exemple, vit avant tout du texte, de son façonnement et de son esprit, la musique n'étant qu'un emballage, un atout de vente?

2.2. Dans cette tradition, Massenet joue un rôle-clef. Grâce à sa place dans la vie musicale de son temps, sa musique ne saurait passer inaperçue, il est écouté et sert de modèle à certains. ${ }^{8}$ Plus que d'autres, il mène à la perfection ce qui avait été amorcé par Gounod tout en tentant une assimilation discrète de ce qu'il a découvert dans Wagner. Il ne sera dépassé en ce domaine que par Debussy. Certaines réserves (peur de l'innovation brusque, désir de plaire au public, goût du succès) l'ont empêché, il est vrai, de transgresser les limites des conventions comme le fera Debussy : le pouls sous-jacent est toujours sensible, la grille rythmique stylise toujours le mètre de la prosodie verbale, il s'agit toujours de musique "versifiée ", et une prose musicale au sens strict du terme n'est atteinte que sporadiquement. Mais Werther, c'est presque Pelléas.

2.3. La déclamation massenétiste écoute attentivement la déclamation théâtrale de l'époque, donc un parler qui reflète, avec une stylisation devenue minime, la prosodie "naturelle " et "idéale " (c'est-à-dire décantée de régionalismes ou de couleurs individuelles). Elle est bâtie, essentiellement, sur des séquences rythmiques dérivées d'un modèle fondamental : le principe de l'anapeste qui s'étend jusqu'à l'anacrouse multiple; le poids ou le temps fort est donc situé à la fin de chaque phase textuelle ou respiratoire. Tous ce qui découle de cette donnée de base va dans le sens d'une traduction subtile de la prosodie française en musique : 
a) Anapestes purs :

Manon, V. $251^{9}$
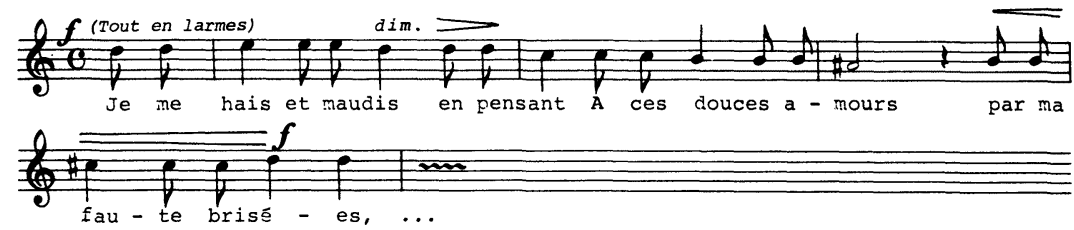

b) Structure anapestique, brèves au rhythme pointé :

Héroidiade, II, 549

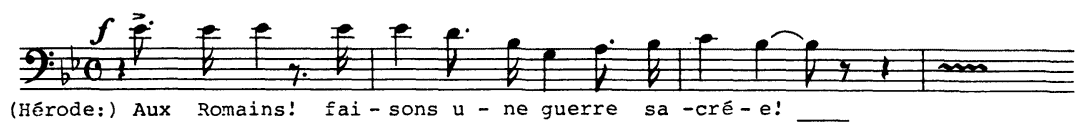

c) Structure anapestique, subdivision tripartite des brèves :

Manon I, 227

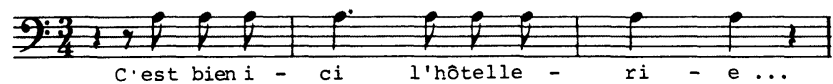

Manon, III/2, 253

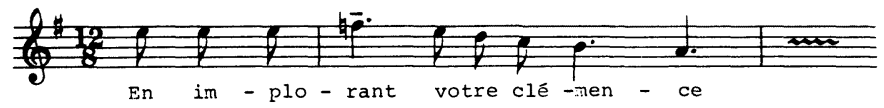

d) Anacrouses multiples :

Werther, III, 469

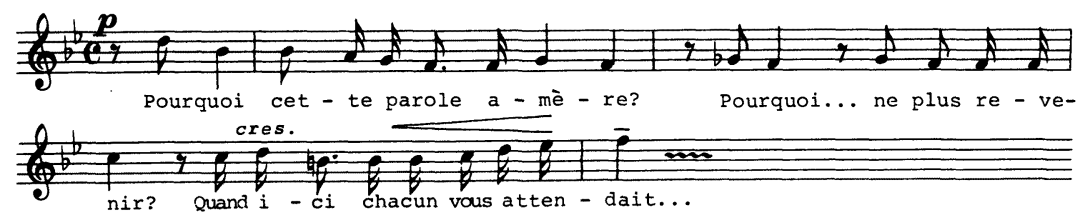


a) Le temps fort initial est souvent évité.

Temps forts évités :

a) Manon, II, 367

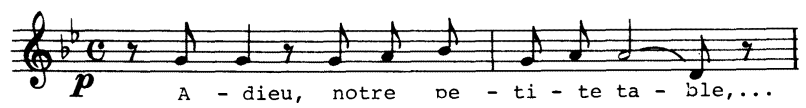

b) Manon, I, 427

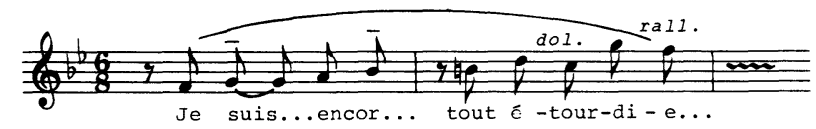

c) Werther, I, 641

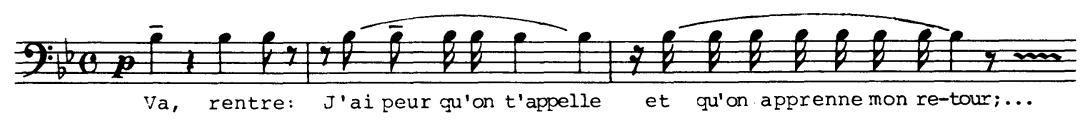

Exemple 2

b) Des syncopes aident à décaler l'accent musical pour éviter qu'un accent lexical (ou accent "tonique», terme peu pratique) coïncide trop régulièrement avec un temps fort musical ; cela est fonction de la réalité prosodique française qui marque très faiblement les accents lexicaux à l'intérieur d'une phase respiratoire.

Distribution syncopée du chant :

a) Manon, II, 267

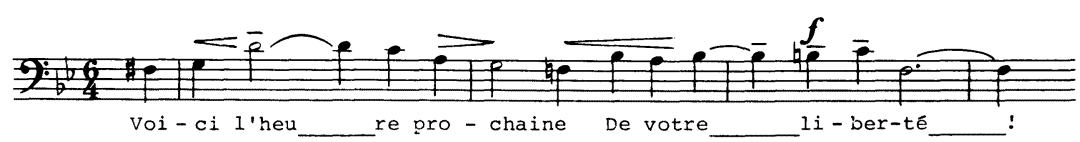

b) Hérodiade, I, 742

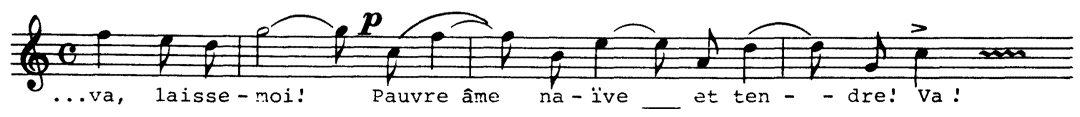

Exemple 3 
c) Les règles concernant la liaison et le e caduc sont soigneusement observées.

Traitement du e caduc :

Werther, II, 302

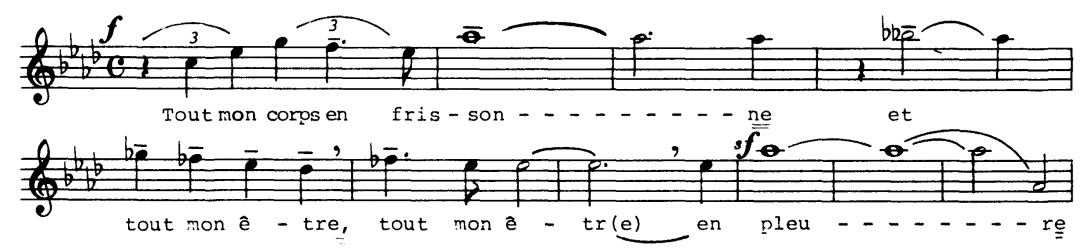

Exemple 4

d) Les fins de phases sont - comme dans la prosodie verbale souvent allongées ou marquées par la suspension (légère ascension de la voix, caractéristique de l'intonation française).

Suspension :

a) Werther, II, 682

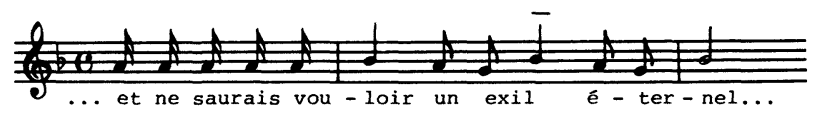

b) Werther, III, 76

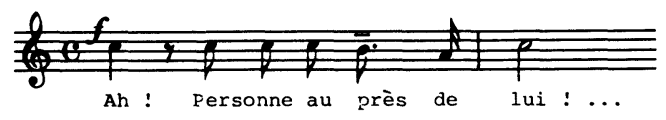

c) Manon, II, 374

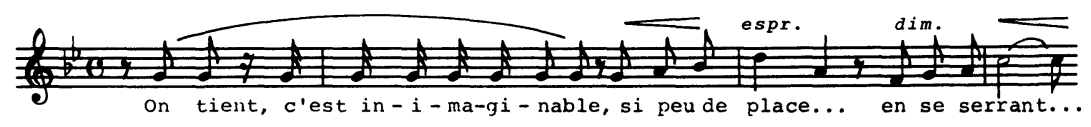


e) Sauf dans les airs visant à mettre en valeur les capacités vocales (et qui subsistent malgré tout), on observe des lignes mélodiques à amplitude assez restreinte et aux ondulations " souples ", reflet musical de l'intonation langagière.

Ligne à petite amplitude :

Thaïs, I/2, 411

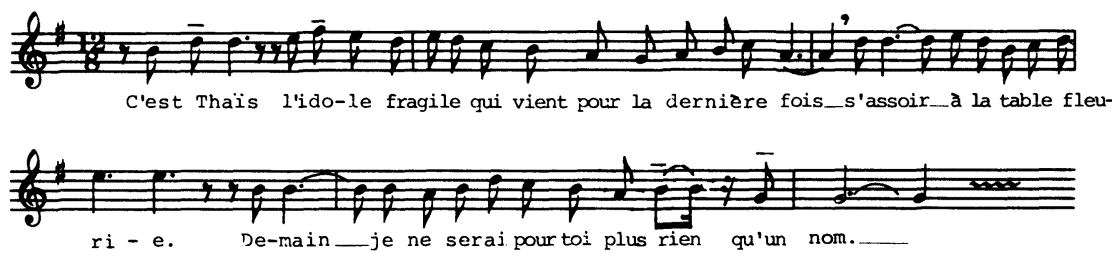

Exemple 6

f) Le jeu des différents types d'accent d'intensité (lexical, de groupe, emphatique, affectif etc.) est rendu de façon très nuancée et vient raffiner la régularité métrique. Pour traduire l'opposition fort/faible (la longueur n'étant marquée en français que de manière instable), la musique tient à sa disposition l'accent exprimé par le temps fort ou demi-fort et l'allongement de la durée, éléments qui se trouvent souvent doublés ou renforcés par les effets des hauteurs (fréquences). Le compositeur utilise en plus les signes de tenuto et marcato, ainsi que les maintes possibilités de décalage rythmique pour saisir à la fois le jeu des différents accents avec leur marquage gradué et leur réalisation souple dans la prosodie « naturelle» .

Jeu de différents accents :

Werther, I, 790

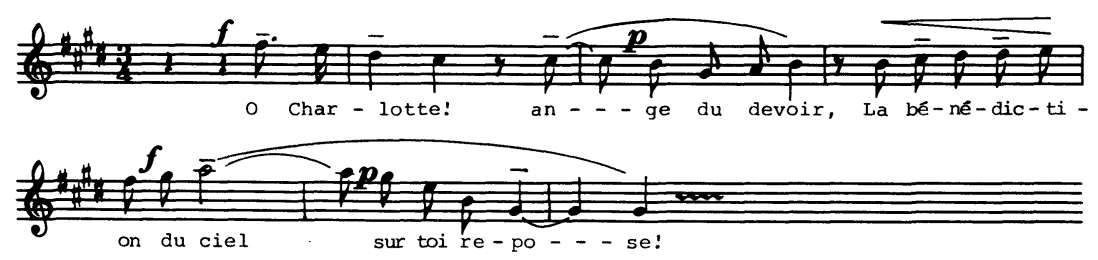


2.4. La place manque ici pour montrer en détail ce qui, chez Wagner, semble préfigurer cette conception. On peut citer le traitement du langage versifié dans le Ring des Nibelungen, la variété des parlers musicaux dans les Meistersinger von Nürnberg ou encore cet effort pour échapper à la monotonie métrique du texte versifié à l'aide de subtiles variantes rythmiques telles qu'on les relève, par exemple, dans Tristan und Isolde. Il est cependant plus aisé, puisque plus palpable, de démontrer en quoi Wagner est différent : tout est dans le fait que sa mise en musique s'applique à la langue allemande dont l'image sonore est quasiment opposée au français. En outre, Wagner préconise toujours le vers, rimé ou allitérant, pseudo-médiéval ou autre, et par conséquent un artifice ; le vocabulaire souvent archaïsant ne peut que confirmer ce jugement. Le chant allonge notablement les durées " naturelles", c'est-à-dire ralentit le débit par rapport au parler " normal ». En revanche, chez Massenet, on observe la tentative de sortir des contraintes du vers en faveur de la prose rythmée (voir Thaïs) et de ne pas trop écarter le tempo chanté du tempo parlé.

Wagner : Tristan und Isolde, "Isoldes Liebestod"

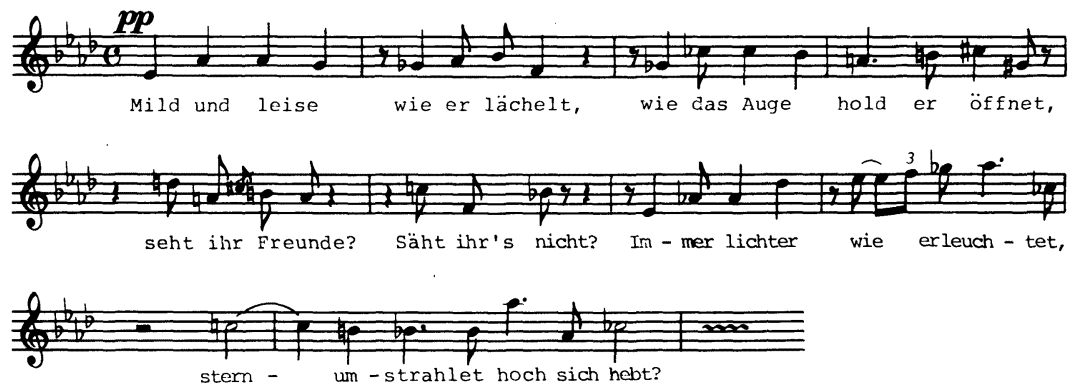

Exemple 8

2.5. Une fois fixés les paradigmes de la déclamation massenétiste, il est indispensable de se tourner plutôt vers l'avenir, vers Debussy, qui s'est avéré " démarqueur " ${ }^{10}$ de Massenet, et cela le plus ouvertement dans L'Enfant prodigue. Sa prosodie musicale se révèle continuation directe des propriétés citées ci-dessus. Mais c'est avec Pelléas que Debussy dépasse définitivement les acquisitions de Massenet, en enfreignant la barrière qui contenait encore la musique de son prédécesseur. Ce qui était présent, dans Massenet, de façon tendencielle et potentielle, devient visible et 
effectif : le cadre métrique s'efface du plus en plus, la prose rythmée prend la place du vers, les valeurs de durées sont découpées asymétriquement (duolets, triolets, quintolets etc. en alternance), la courbe mélodique est neutralisée comme phénomène musical (le résultat étant un retour à une quasi-psalmodie) ou bien devient ligne d'intonation. C'est seulement l'existence de Schönberg qui nous fait hésiter à parler de Sprechgesang face à une prosodie musicale si fidèle.

a) Debussy : L'Enfant prodigue, "Danse"

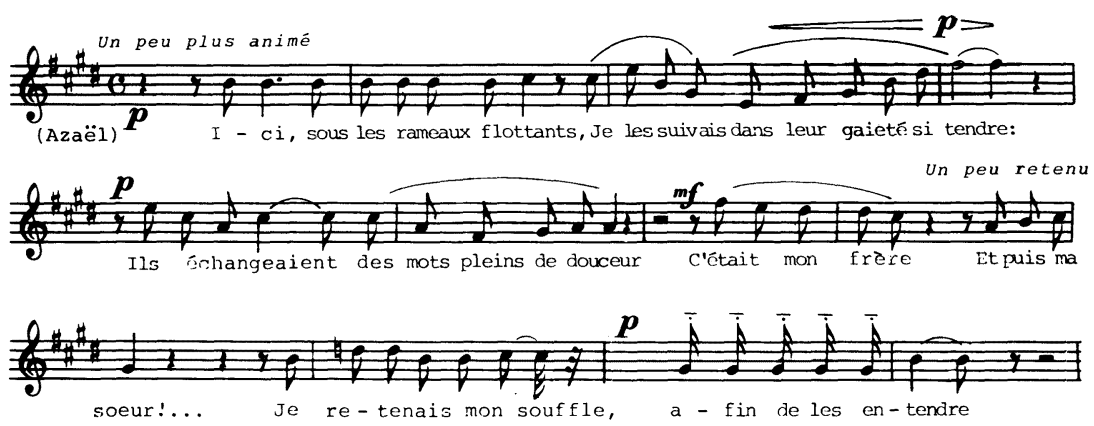

b) Debussy : Pelléas et Mélisande, I/2, 14 (découpage irrégulier du rhythme) :

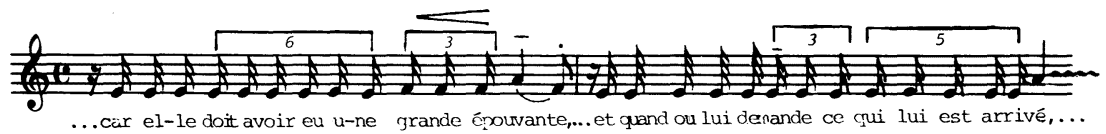

Exemple 9

2.6. Pour prévenir des malentendus, citons toutefois le cas de Carmen. On peut se demander pourquoi Carmen, où la prosodie naturelle est tout simplement caricaturée par la musique, a largement supplanté, sinon Pelléas, au moins les autres opéras de l' "école française ", et ce, sur le plan international. La réponse est partiellement contenue dans la question. L’appréciation générale, voire internationale, est sans doute due aux qualités musicales musique dérivée de danses folkloriques stylisées - indépendantes du texte, ainsi qu'au thème central de l'action : l'érotisme égoïste de la femme fatale (voir la reprise du sujet par le cinéma). Il semble évident qu'un opéra qui observe minutieusement la prosodie et s'interdit le panache, fait plus ésotérique qu'une oeuvre qui recherche le geste musical intuitivement saisissable. Mais 
rappelons aussi qu'un sujet proche de celui de Carmen a été traité, par Charpentier, dans Louise, où la prosodie est observée avec minutie, voire calquée de façon naturaliste. ${ }^{11}$ Et Louise a fait recette comme Carmen! Mais de façon générale, une oeuvre qui dọit beaucoup au lien avec sa "langue maternelle " est non seulement difficile à dissocier de ce lien mais semble susceptible de rester attachée au cadre national (voir les difficultés d'appréciation que rencontre jusqu'aujourd'hui Pelléas dans les pays germanophones).

2.7. Sur le plan de la mise en musique du texte, nous pouvons donc reconnaître un certain nombre de paradigmes qui se présentent tantôt comme formules, configurations ou dérivés de formules (anapeste, suspension etc.), tantôt comme types d'agencement (temps fort évité etc.) et dont chacun a une histoire et une origine souvent situées à l'extérieur de la musique. L'alignement et la combinaison de ses paradigmes relèverait alors de l'analyse syntagmatique.

3. L'analyse des constituants proprements musicaux - qui remontent à des données acoustiques - révèle aussi bien des analogies que des contrastes par rapport au façonnement de la déclamation musicale.

3.1. Il a toujours été souligné que Massenet est avant tout mélodiste, sans que l'on ait mis en valeur les conséquences de cette option. Dans l'ensemble, on peut dire que la " souplesse " retenue comme caractéristique de sa mélodie tire son origine, en grande partie, du traitement musical de la langue : c'est ce style vocal aligné sur la prosodie française qui déteint sur la conception de la mélodie instrumentale. Cette conception s'oppose à la tradition, tout à fait notoire dans l'opéra, de prendre la voix pour un instrument et de lui demander des techniques et des exploits dérivés des possibilités instrumentales. ${ }^{12}$ Elle s'oppose, au niveau même de l'écriture, à une tradition mélodique fondée sur des critères intra-musicaux de stylisation : enchaînements de rythmes fixes, pointés ou autres, rythmes de danse, formations rythmiques ciselées selon des calculs de durées abstraits, structure intervallique dérivée d'une pensée harmonique fonctionnelle ou de sequences abstraites de hauteurs etc. Face à des types de mélodie marqués par la fermeté, la tension et le dynamisme internes, au profil plutôt acéré et anguleux, le melos massenétiste ainsi que sa prolongation chez Debussy se distinguent par leur aspect statique, détendu, par une certaine " lassitude ". ${ }^{13}$

3.2. En regardant de près la mélodie de Massenet, on constate 
qu'elle est composée, au niveau paradigmatique, d'un certain nombre de cellules stéréotypées et de structures récurrentes qui, outre leur rapport avec les éléments prosodiques, semblent renvoyer tantôt à des traditions françaises, tantôt à des formations archétypiques :

a) Massenet a visiblement une prédilection pour les structures tripartites. La fréquence de mesures ternaires, simples ou composées, en témoigne autant que maint motif mélodicorythmique ou certains procédés de développement formel. Quant au rythme et au paramètre de la durée, on relève souvent la relation $2: 1$, réalisée par groupes répétés de deux notes, ce qui fait penser au principe de l'inégalité de la musique baroque française.

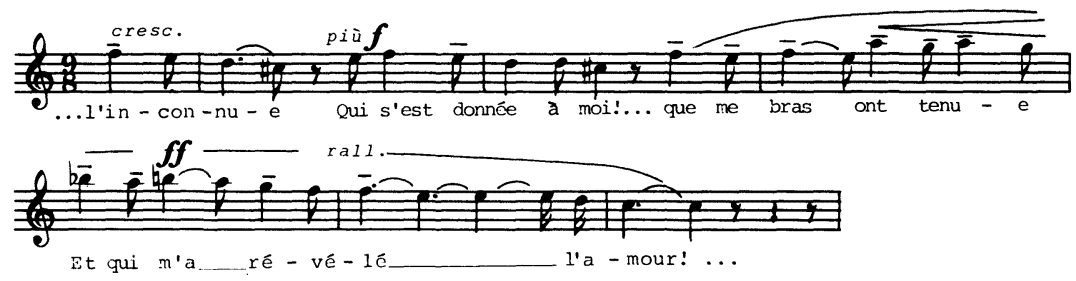

Exemple 10

b) La subdivision ternaire ou autrement impaire, génératrice d'un sentiment de rotation et susceptible d'atténuer le contraste fort/faible, est souvent combinée avec la subdivision binaire, soit par alternance, soit par superposition. De telles alternances font davantage sentir la durée de l'unité sous-jacente, voir de la mesure entière, où elles effacent même parfois le poids du temps fort initial. Elles vont donc dans le sens, à la fois, d'un flottement métrique et d'une respiration par phases longues. 
a) Ariane, III, 824

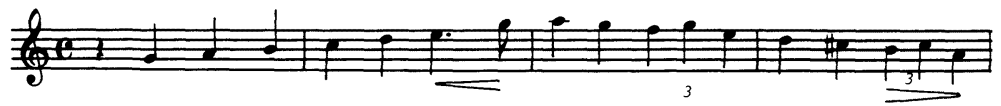

b) Werther, II, 564

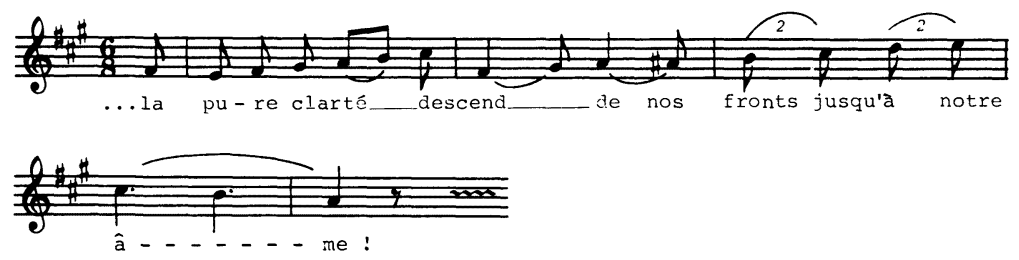

Exemple 11

Si les subdivisions variables sont progressivement développées par les compositeurs du XIX ${ }^{\mathrm{e}}$ siècle, ${ }^{14}$ il faut dire que, en France, c'est avec Debussy qu'elles atteindront un niveau de différenciation particulièrement élevé.

3.3. Un certain statisme mélodique est dû, en outre, à deux types de paradigmes :

c) La mélodie tourne souvent autour d'un axe horizontal (ce qui n'est pas sans rappeler les mélismes orientaux) ou bien se meut entre deux axes, sorte de tangentes faisant penser à la quinte fixe de la cornemeuse.

Staisme mélodique, axe horizontal :

a) Werther, Prélude, 17

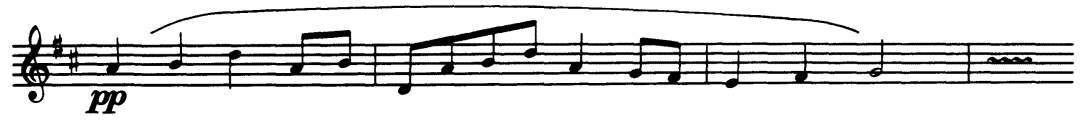

b) La Vierge, "Prélude pastoral "

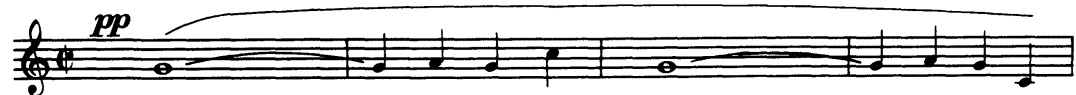

Andantino mod.

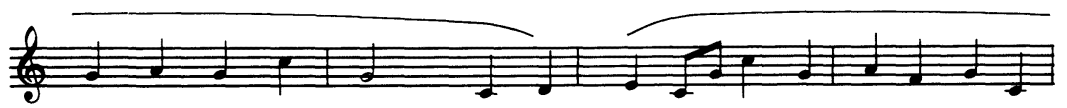


d) Ce même principe se retrouve, "en miniature " pour ainsi dire, au niveau des particules mélodiques : celles-ci ont souvent la forme de "fioritures figées " ou d' " ornements ralentis " qui ont perdu leur statut d'accessoire pour devenir des constituants mélodiques. Il s'agit de séquences en forme de tour de gosier ou de broderie, l'intervalle de seconde propre à ses fioritures pouvant s'étirer jusqu'à la quinte, quarte, sixte, et la figure elle-même étant bâtie sur un axe horizontal, sa base linéaire.

Fioritures figées intégrées au cours mélodique :

a) Manon, I, 246

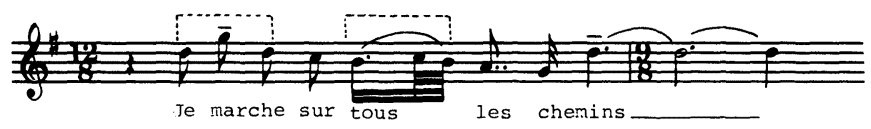

b) Le Cid, Ouverture

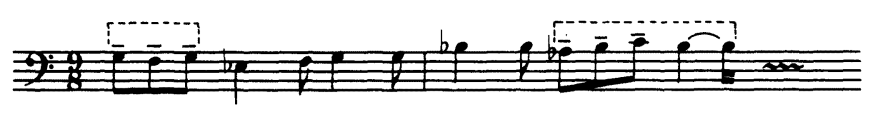

Exemple 13

3.4. C'est avec une petite cellule ressemblant à un mordant figé (par exemple : la suite mi-ré-mi) que l'évocation d'archétypes musicaux semble se justifier. On pourrait y voir l'incarnation minimale du mouvement mélodique marqué par le statisme : son de départ - écart - retour au son de départ. Il y a donc à la fois marche mélodique et présence d'un axe horizontal. Sans parler du chant grégorien et des chants des troubadours, on trouve les variantes de cette cellule à maintes reprises dans la musique du Moyen-Âge, de la Renaissance, jusqu'à l'époque baroque (toujours en dehors des ornements proprement dits, bien entendu), comme en témoigne un petit extrait du $20^{\mathrm{e}}$ motet de Guillaume de Machaut ${ }^{15}$ ou encore le début du mouvement lent du Concerto italien de J.S. Bach. Elle n'est pas absente des musiques d'autres époques et réapparaît en particulier là où est fait allusion, par imitation, évocation ou citation, à la musique baroque : à vérifier dans le Concierto de Aranjuez de Joaquin Rodrigo, écrit en 1939, où le début de l'Adagio semble se référer au motif de Bach. Notons que même chez Massenet, des cellules semblables apparaissent lorsqu'il est question du XVIII ${ }^{\mathrm{e}}$ siècle, voir le motif du menuet de 
Manon ou avec une fréquence frappante dans le contexte religieux, biblique ou archaïque.

Archétype mélodique chez Guillaume de Machaut :

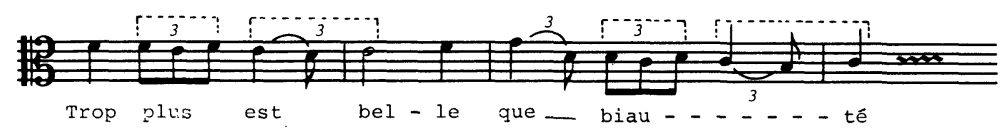

Exemple 14

Manon, Menuet

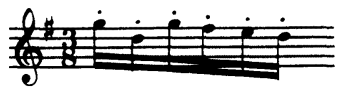

Exemple 15

a) La Vierge, $n^{\circ} 4$, «Duo"

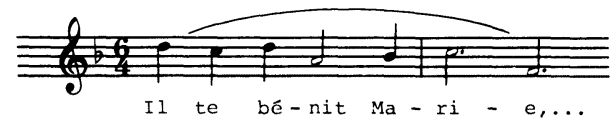

b) Hérodiade, I, 246

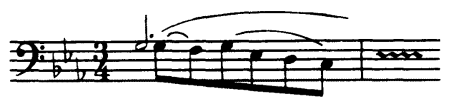

Exemple 16

3.5. Il serait important de retracer l'historique de ce genre d'archétypes à travers les styles et époques, ce qui ne saurait être fait ici ; les résultats d'une telle recherche nous apprendraient sans doute beaucoup sur l'invariabilité de certains faits musicaux, même très concrets. Pour nous, il suffit de souligner que différents types figés de broderies et autres fioritures, répétées, transposées, mises en série et variées, constituent un trait essentiel, également, du melos debussyste et le rapprochent de l'arabesque, terme effectivement utilisé, comme on sait, en rapport avec sa musique. 
Debussy : Pelléas et Mélisande :

a) Prélude, 5-6

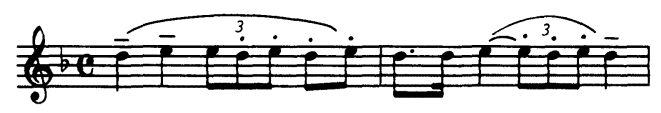

b) Prélude, 18-19

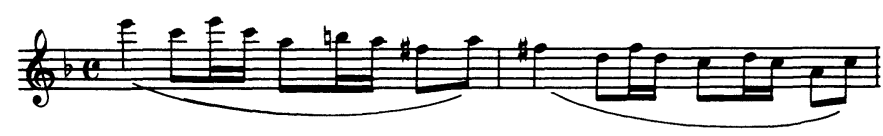

Exemple 17

Chez Puccini, dont le lien avec l'opéra français de la fin du $\mathrm{XIX}^{\mathrm{e}}$ siècle n'a jamais été assez démontré, on relève des formules analogues, intégrées au style élégiaque et dramatique qui lui est propre. Et c'est Puccini aussi qui semble adopter, au point d'en faire un élément de son image de marque, cet autre petit motif massenétiste, formation " triangulaire " qui existe en une multiplicité de variantes mais dont certaines - quatre ou cinq environ - deviennent de véritables topoi, lyriques ou dramatiques selon le cas.

Puccini : Madame Butterfly

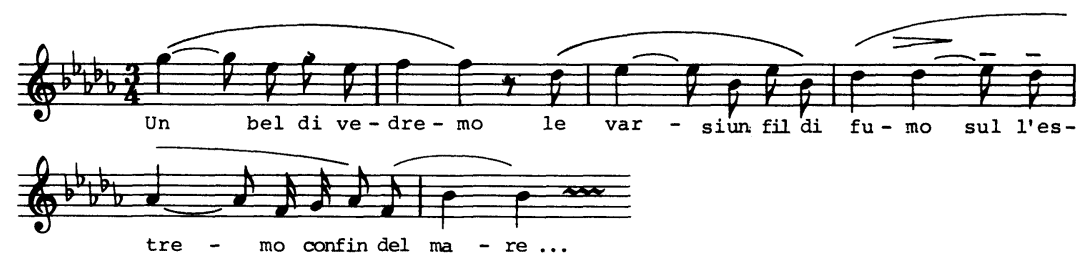

Exemple 18 
Motif « triangulaire "

a) type statique lyrique

Werther, Clair de lune

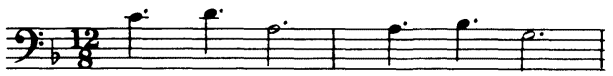

b) type dramatique

Esclarmonde, Prologue, 90

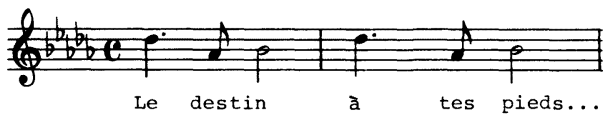

c) type pathétique, élégiaque

Manon, IV, 246

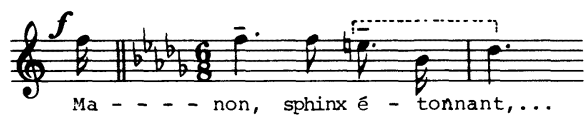

d) en fonction de suspension

Cendrillon, III/3, 27

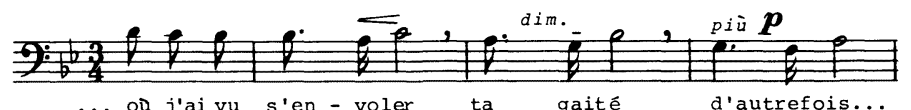

Exemple 19

4. De quelle façon ces éléments sont-ils assemblés pour donner des mélodies, des formes? Quelles sont les propriétés syntagmatiques du langage massenétiste?

4.1. La plupart des techniques de développement employées par Massenet frappent par leur caractère simpliste et stéréotypé. Apparemment réfractaire au travail symphonique, Massenet connaît tout de même des procédés fondés sur le motif, le recours à des motifs de rappel ou à des motifs-symboles. Il vaut mieux éviter le terme leitmotiv car, malgré le rayonnement de Wagner auquel n'échappe pas Massenet, ce serait une source de malentendus. Hormis Werther où le motif-conducteur a le plus évolué et 
détermine souvent le déroulement dramatique, Massenet l'utilise d'une manière beaucoup moins stricte, moins fréquemment, et le varie très peu. Le motif sert rarement de pierre à bâtir toute une scène ou de noeud déterminant la structure formelle, bien au contraire : même là où la mise en musique intégrale au sens wagnérien du terme est visée, la forme reste assez classique et segmentée, il y a des passages contrastants ainsi que des morceaux et des airs de forme close. Si, à la limite, un rapprochement du premier Wagner paraît possible, ce besoin de transparence, cette peur d'effacer le " plan ", d'exposer le spectateur-auditeur à des risques d'égarement, semble correspondre avant tout à un besoin " latin » de clarté, besoin conscient ou inconscient mais en tout cas omniprésent et jusqu'à nos jours pilier principal de tout enseignement, de toute culture française : la simplicité n'est pas un vice, la complexité, pas une vertu. Il ne faut pas perdre cela de vue - ni le fait que Massenet écrivait pratiquement toujours, et parfois un peu trop, pour le public - lorsqu'on veut juger les procédés de construction du compositeur.

4.2. La répétition comme principe formateur : voilà qui est presque une banalité. Or, la répétition littérale, avec un minimum de variation, est légion chez Massenet. On repère toutefois des manifestations bien distinctes de ce principe :

a) Il s'agit normalement d'un simple dédoublement (de cellule, de motif, de phrase, de thème), l'inversion ou la forme rétrograde étant extrêmement rares, ainsi que la diminution ou l'augmentation des valeurs.

b) S'il y a transposition, celle-ci se réduit souvent à la rosalie (fréquente déjà chez Gounod) ou à une répétition n'impliquant que les modifications minimes et nécessaires dues au changement de degré à l'intérieur de la tonalité donnée; sur le plan de la répétition immédiate, le changement de caractère (majeur/mineur, modulation, contraste harmonique) est plutôt l'exception. 
a) Esclarmonde, Introduction, 15

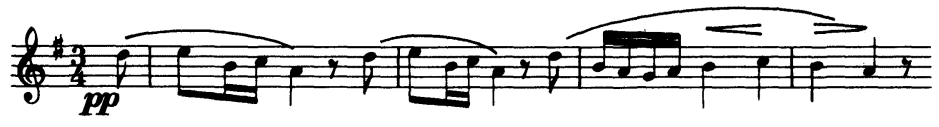

(dédoublement de motif sans et avec modification)

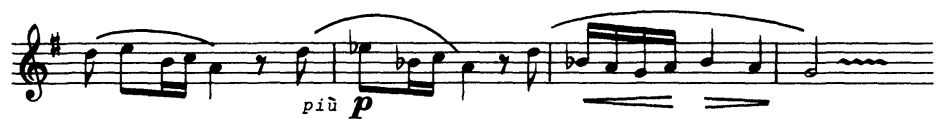

b) Marie-Magdeleine, III, $\mathrm{n}^{\circ} 13,18$ (rosalie)

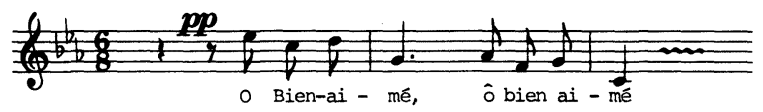

c) Panurge, II, 336 (transposition tonale)

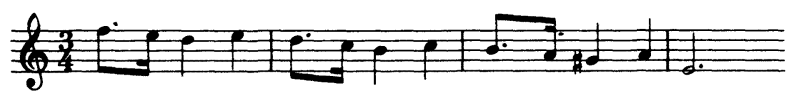

Exemple 20

L'unité répétée $(A)$ est suivie tantôt d'une continuation libre (X), tantôt d'une partie constituant un pendant de $A+A$ (donc $B$ ) et souvent même bâtie avec le matériel de $A\left(B_{A}\right)$. Nous avons alors affaire au schéma $A-A-B$ (ou $A-A^{\prime}-B$ ou $A-A^{\prime}-B_{A}$ etc...) qui se retrouve fréquemment à tous les niveaux de formes. ${ }^{16}$ C'est la fréquence qui justifie de parler de schéma typique et de forme stéréotypée. Le même schéma se retrouve, certes, chez d'autres auteurs, mais il serait impossible de retracer toutes ces occurrences et toute son évolution, d'autant plus qu'il est difficile de dire jusqu'à quelles limites de variation et d'étendue il est supposé l'identifier comme tel. Alfred Lorenz l'a même reconnu dans la macrostructure des oeuvres de Wagner. Toujours est-il que le schéma A-A-B remonte à la très ancienne Barform, caractéristique des troubadours et des maîtres-chanteurs. ${ }^{17}$ Et le dédoublement ainsi que la forme A-A-B sont intéressants pour nous parce que Debussy a de toute évidence une prédilection semblable pour ces procédés. ${ }^{18}$ 
La Navarraise, I, 1

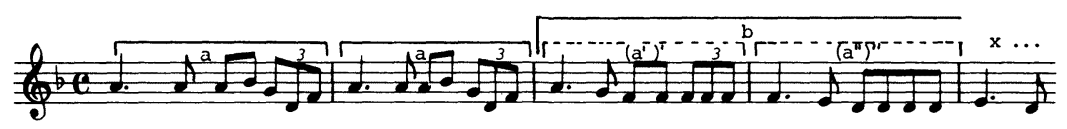

(Voir aussi l'exemple 20a)

Exemple 21

5. L'harmonie joue souvent chez Massenet un rôle particulier. En principe, il reste fidèle au système tonal et à l'harmonie fonctionnelle, mais on voit s'amorcer des techniques susceptibles de contrecarrer, voire de contourner ou de dépasser l'engrenage fonctionnel. Ces techniques sont toutefois ancrées dans de très vieux procédés, pré-fonctionnels pour ainsi dire, et comme dans les autres domaines, Massenet ne les introduit que prudemment, n'en fait pas l'essentiel de son langage. Ce sera là le privilège de Debussy. Néanmoins, la porte est entrouverte.

5.1. Retenons tout d'abord qu'il est vain de chercher des affinités avec Wagner. La présence de chromatismes ne doit pas nous tromper. Chez Wagner, la progression harmonique, y compris le chatoiement chromatique jusqu'à la plus parfaite ambiguïté, constitue la trame du développment musical dont découlent les autres facteurs, notamment la mélodie et l'instrumentation. Chez Massenet, le chromatisme a plutôt une valeur d'accessoire, de complexité passagère, voire de coloration, contrebalancée par la détente et la simplicité harmoniques. Le chromatisme y apparaît davantage comme formant mélodique et marque certains motifs ou peintures sonores. Plus que le chromatisme à l'impact wagnérien, c'est l'enharmonie et les rapports par tierces qui comptent pour Massenet et par lesquels il pousse très loin la notion de fonctionnalisme. Parfaitement conscient des transitions, des ambiguïtés et des absurdités que permet le système fonctionnel, il semble parfois vouloir les démontrer par des "jeux harmoniques" qui, bien que rappelant des exercices de conservatoire, sont mis au service d'une expression précise. Bien entendu, tout cela reste encore "définissable » . 
Thaïs, II/2, 15

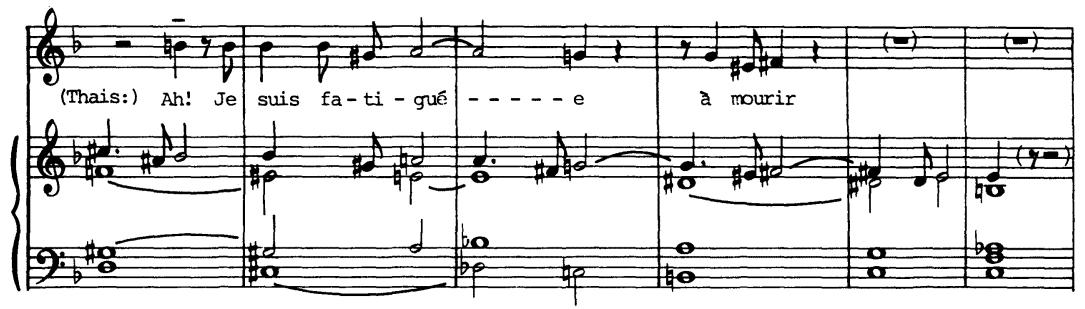

Exemple 22

a) Werther, II, 716

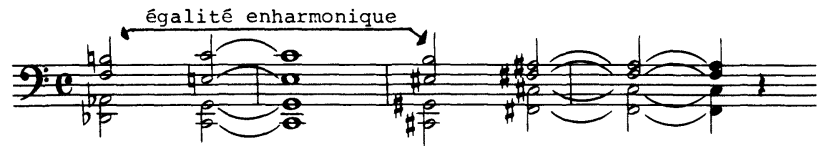

b) Arianne, II, 198, 204, 208

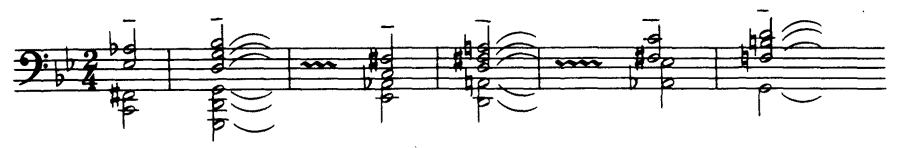

Exemple 23

5.2. Massenet dispose des acquis harmoniques du $\mathrm{XIX}^{\mathrm{e}}$ siècle, y compris des accords de neuvième et de l'engrenage des dissonances. Mais on note généralement une certaine peur des âpretés (sauf dans Werther), une préférence des dissonances " molles ». Celles-ci vont des accords de septième mineure (avec tierce majeure ou mineure), parmi lesquels - évidemment, puisque chargé d'une connotation "française " - l'accord de la sixte ajoutée, jusqu'à des formations ressemblant déjà aux étagements de secondes que l'on retrouvera chez Debussy. Soulignons à nouveau que, chez Massenet, de tels agrégats peuvent toujours se ramener au système tonal. Ce qui est original, c'est moins la composition des accords, qui reste plutôt traditionnelle, que leur enchaînement. C'est là une réalité aussi simple que fondamentale, d'autant plus qu'elle nous semble caractéristique de 
la manière française de concevoir des innovations, caractéristique également de l'harmonisation et de l'image sonore debussystes.

a) dissonances molles :

Werther, II, 725

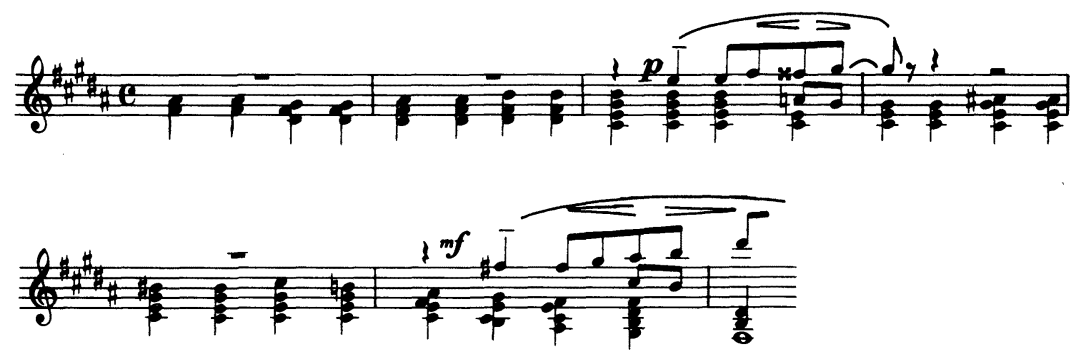

b) étagement de secondes :

Manon, II, 366

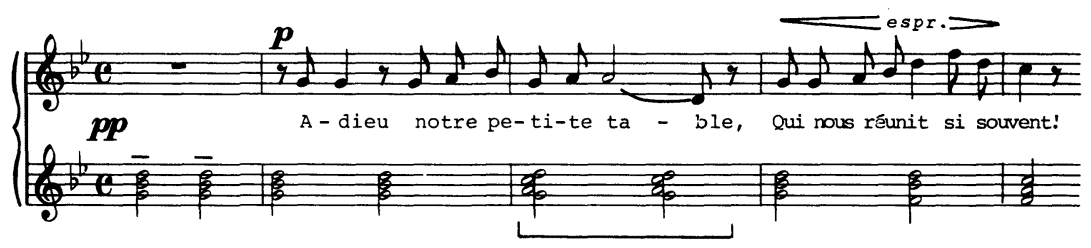

Exemple 24

5.3. Structures verticales plutôt traditionnelles : cela signifie aussi que certaines configurations ressenties commes nouvelles et significatives chez Debussy, ou portant chez lui l'étiquette de "valeur harmonique indépendante", ont chez Massenet un curieux statut transitoire : ils sont encore intégrés ou intégrables au système fonctionnel, mais se trouvent en voie de détachement. Le meilleur exemple en est sans doute l'accord de quinte augmentée ou, plus exactement, le "système par tons " (accords formés à partir de la gamme par tons). En principe, la totalité des configurations verticales résultant de la gamme par tons peuvent être réinterprétées comme accords dérivés du système tonal et fonctionnel (voir tableau ci-joint), à condition de se limiter à 
Ce tableau ne présente que les formations bâties sur le son inférieur de la gamme ; les transpositions mènent à des résultats analogues.

Les formations harmoniques sont classées par groupes de renversements et sont données en version déployée.

\section{gamme départ :}

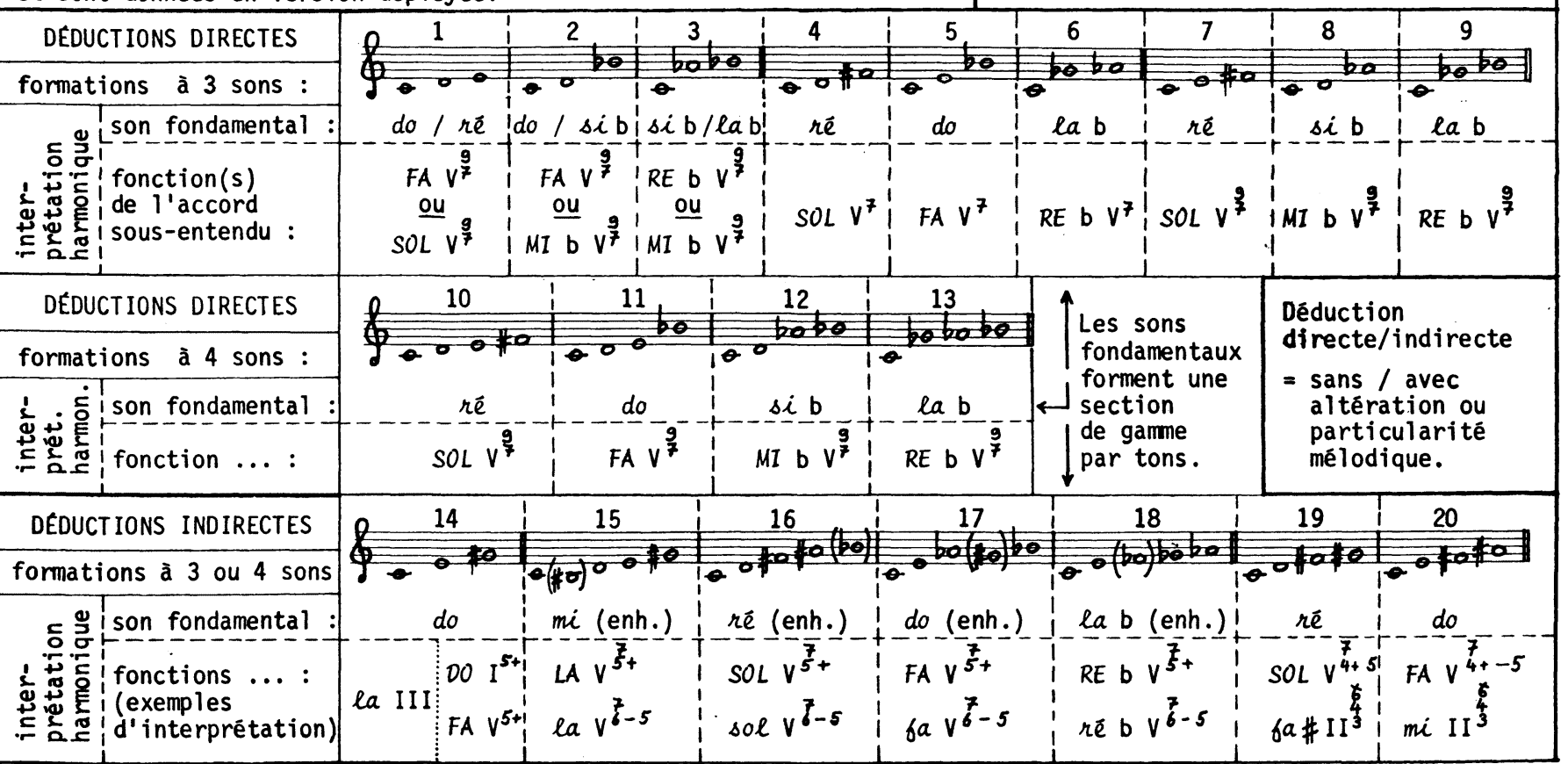


quatre sons. ${ }^{19}$ Le " renversement " et le contexte harmonique choisis préjugeront alors de l'intégration tonale possible. C'est exactement ce que nous trouvons dans Massenet par rapport à Debussy. Alors que, pour Debussy, le système par tons se sera émancipé comme mode équivalent à côté du système tonal, avec cette sonorité molle, feutrée, instable qui lui est propre dans l'horizontal comme dans le vertical, Massenet ne lui permet pas encore de franchir le seuil de l'émancipation. De tels accords sont ancrés dans un contexte fonctionnel, apparaissent devant un fond servant de repère tonal, alors que leur spécificité sonore est parfaitement saisie et mise en valeur.

a) exploitation dramatique d'une section de gamme par tons :

Werther, II, 692

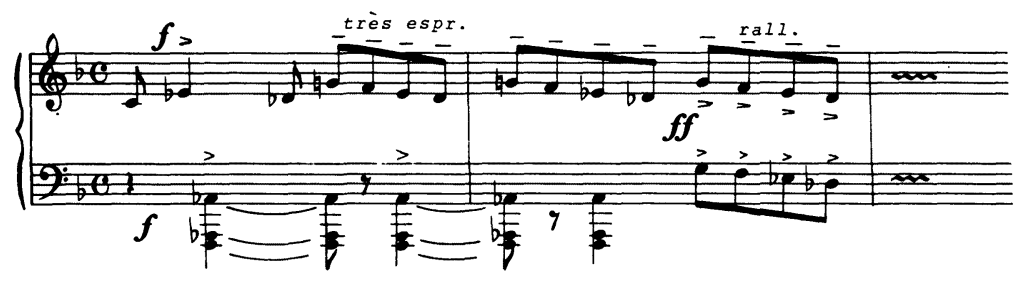

b) apparition, dans une modulation, d'accords coïncidant avec le système par tons :

Manon, V, 169

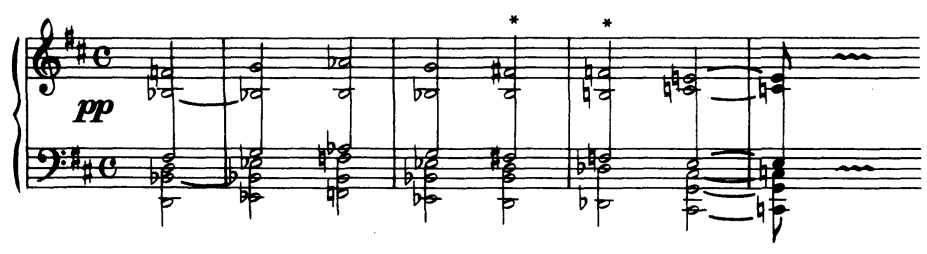

Exemple 25

Naturellement, plusieurs facteurs rendent possible la double interprétation (appartenance au système tonal ou à un mode indépendant) : la gamme par tons coïncide partiellement avec des sections d'autres modes (sons 4 à 7 de la gamme majeure, sons 1 à 4 du mode lydien, sons correspondants dans les autres modes ecclésiastiques, sons 3 à 7 de la gamme mineure à la sixte et à la septième augmentées) ; les agrégats en question ne comprennent 
ou parcourent généralement pas plus de quatre sons différents et ne constituent par là qu'une représentation partielle, incomplète, de l'accord tonal sous-entendu et de la gamme par tons; ils sont ressentis plutôt comme écarts par rapport à la norme tonale du contexte. Mais d'autre part, leur valeur sonore s'apparente aux autres effets sonores importants chez Massenet : préférence des dissonances molles, groupements de secondes majeures, rapports fondés sur la tierce. Quant aux autres modes figurant occasionnellement dans les partitions, leur intégration ou dérivation est tout à fait analogue : ils apparaissent comme des écarts de la gamme majeure/mineure la plus proche.

5.4. Originalité de l'enchaînement : voilà comment la syntaxe harmonique de Massenet devient réellement significative puisqu'elle s'avère liée au primat mélodique. En effet, la pensée mélodique, le principe de la linéarité, semble empiéter sur le terrain de l'harmonisation. Outre les enchaînements traditionnels, on observe deux tendances, liées l'une à l'autre. D'une part, le compositeur établit souvent "à grande distance" ses piliers harmoniques. Autrement dit : il pose une harmonie de base qui reste constante pendant une assez longue période, parfois sous forme de pédale ou d'accord tenu; au-dessus, la succession de sons ou d'accords fait fonction d'une simple fioriture mélodicoharmonique. D'autre part, certaines successions d'accords apparaissent comme des juxtapositions a-fonctionnelles de valeurs sonores. Il peut s'agir d'un accord fixe et graduellement décalé, harmonisant comme une mixture d'orgue la ligne mélodique (ce qui est un cas d'espèce de la fioriture mélodico-harmonique), ou du fameux "balancement harmonique " qui se présente comme l'alternance de deux accords dont la qualité différente ne résulte au fond que d'un effet mélodique minimal : un ou deux sons s'écartent de l'accord de base pour retrouver immédiatement la position originale, et ainsi de suite. Ce n'est en vérité qu'un seul champ harmonique fondé sur un accord fixe (ce qui rejoint le cas précédent : l'harmonie constante) et ne présentant que des variations mélodiques internes définissables comme retards ou anticipations. 
Accords-Mixtures sur pédales :

Manon, III/2, 157

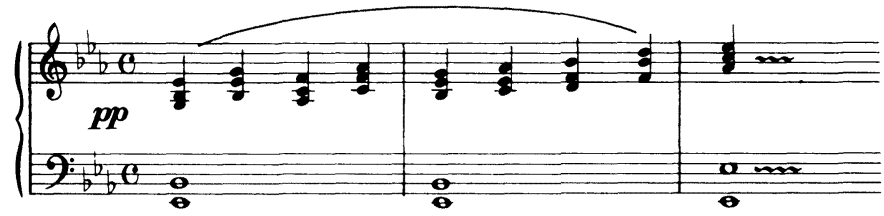

Exemple 26

a) accords décalés sans lien fonctionnel:

Thaïs, II/3, 158

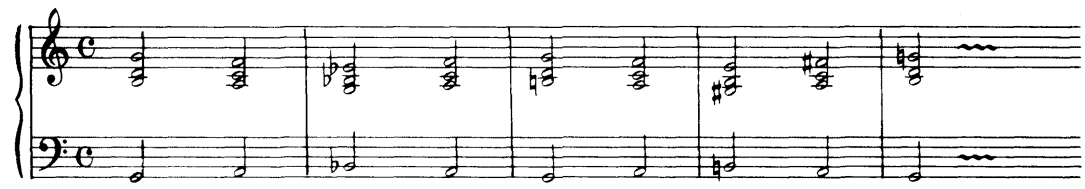

b) balancement harmonique :

Esclarmonde, II, 808
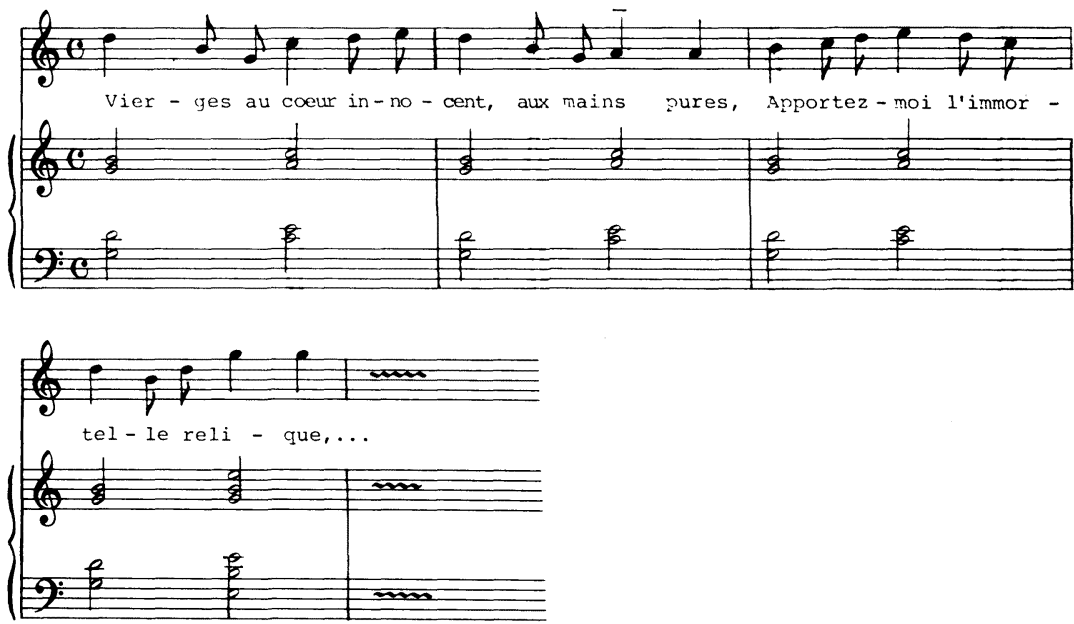
Ce sont là les principales causes d'un certain statisme harmonique qui rejoint nos observations concernant le statisme mélodique résultant de la présence d'un axe horizontal et de lignes rappelant les arabesques. Ne trouve-t-on pas chez Debussy le même type d'harmonisation, réalisée toutefois avec bien plus de rigueur : configurations verticales juxtaposées comme des couleurs apparentées ou contrastantes sans nécessité de lien fonctionnel, soit pour prêter un fond sonore aux arabesques mélodiques, soit pour illuminer de façon chatoyante la mélodie?

5.5. Mais dire que l'harmonie massenétiste est statique ou, pire, monotone, ne serait que la moitié de la vérité, voire un malentendu. Le recours fréquent aux altérations, aux modulations éloignées et aux accords de septième et de neuvième prouve la recherche de variété et d'expression juste. En outre, on relève au passage quelques astuces et âpretés, comme des successions de quintes, de quartes, ou ces superpositions explicables comme "bitonalité avant la lettre ". En particulier, l'harmonisation banale est souvent évitée par le fait que la mélodie semble se situer, par rapport à son harmonisation, dans les sons supérieurs de la colonne des harmoniques, au niveau des quintes, septièmes, neuvièmes, ce qui explique certains effets modernistes et prépare la mélodie éthérée des impressionnistes.

Bitonalité apparente :

Manon, Prélude, 70

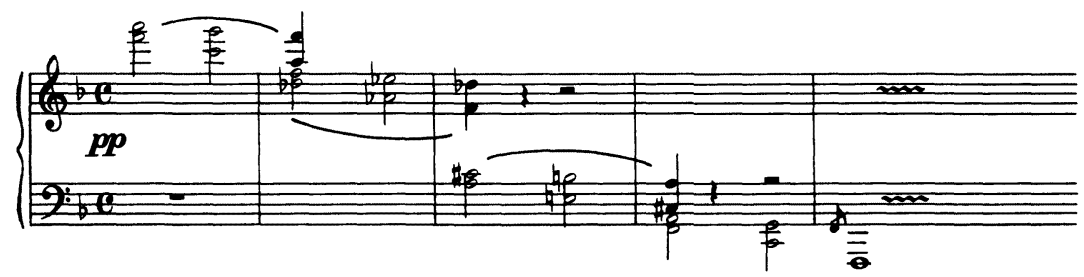

Exemple 28

5.6. Au regard du langage impressionniste, toutes ses observations se centrent autour de deux principes : l'arabesque mélodique qui agrémente le champ harmonique posé, et l'harmonisation comme accessoire mettant en relief par une succession de couleurs l'essentiel : la mélodie. La mélodie épanouie de Massenet, si souvent citée, cette ligne aux courbes équilibrées, un peu lasse et 
non dépourvue de sentimentalité, répond aux deux faces de la médaille : par son allure indépendante, elle se conçoit comme arabesque par excellence et comme événement linéaire essentiel que servent l'harmonie et l'instrumentation. ${ }^{20}$

Et en se tournant vers le passé, on constate que les procédés qui prennent de l'importance chez Massenet et se cristalliseront dans le langage debussyste, renouent avec des styles très anciens. L'harmonisation modale où chaque accord a une valeur plutôt indépendante redevient une source d'innovation chère aux compositeurs français. La pensée linéaire était à la base de la polyphonie et l'on peut vérifier sa prédominance en reculant jusqu'au chant grégorien. Faut-il aussi évoquer les rapports des chants des troubadours avec la musique arabe?

5.7. Nous avons fait allusion à l'instrumentation de Massenet qui mériterait, en effet, une analyse approfondie. Ici, quelques indications doivent suffire pour montrer ses affinités avec les " mixages harmoniques " étudiés et avec le sens de pureté et de transparence des impressionnistes. Massenet aime beaucoup les bois pour dessiner des mélismes devant un fond de cordes ou d'orchestre. Mais ayant opté pour l'opéra et pour la structure monodique, il préconise autant le son spécifique de l'instrument seul, pur, ainsi que la structure orchestrale dépouillée, transparente, au point de réellement l'appauvrir dans certaines de ses dernières oeuvre (Roma, 1912). Toujours est-il que, pour l'instrumentation, il prenait pour modèle Mendelssohn, Berlioz et Thomas, non sans se laisser influencer par le Wagner des couleurs tamisées. Si l'on fait abstraction de son épisode vériste ( $L a$ Navarraise et Sapho), on doit avouer que les ingrédients instrumentaux sont toujours savamment mélangés, sans surcharge ni violence, et révèlent une recherche de saveur, de suavité, voire de volupté, qui répond parfaitement aux couleurs harmoniques. Peut-être les comparaisons avec Manet, subjectives et exagérées à première vue, ne sont-elles pas si fausses !

6. Le découpage de la chaîne musicale en paradigmes et l'attribution, à ces paradigmes, d'indicateurs de fonction diachronique nous amène aussi sur le terrain de la sémantique musicale. Ne faut-il pas considérer le dit indicateur comme élément de la composante sémantique du paradigme ? La définition de la "signification musicale " a toujours posé des problèmes aux théoriciens. De la négation polémique d'un Stravinsky au néomysticisme de l'avant-garde autour de Stockhausen, en passant par l'attitude descriptive ou évocatrice de la musique à program- 
me, nous avons vu toutes les facettes d'une mise en rapport avec des phénomènes extra-musicaux, sans être arrivés à des définitions précises. L'accouplement fixe de signifiant et signifié à l'instar de la linguistique étant inadéquate en musique (même les signifiés de la rhétorique musicale baroque ne sont valables que temporairement et pour une communauté restreinte), le sens musical semble se constituer en trois étapes : a) par dénotation (qui ne semble possible que sur le plan intra-musical, comme le sens grammatical en langue), b) par association/similitude/ analogie (où entreraient les dessins de la musique à programme, la description et " l'onomatopée »), c) par connotation. Ainsi, le petit extrait de Werther de notre exemple 25 dénoterait : section de gamme par tons en mouvement descendant répété, intégré à famineur, ici sixième degré de la-b-majeur ; il signifierait par analogie : l'aspiration inexaucée de Werther etc... ; il connoterait : rôle harmoniquement ambigu de la section de gamme par tons dans un contexte chromatique de conception mélodiques; expression d'une esthétique française influencée par des principes wagnériens et s'ouvrant sur l'impressionnisme ; assimilation du sentimentalisme du Sturm und Drang par un sentimentalisme de fin de siècle ... Il semble donc tout à fait possible et utile de faire entrer nos indicateurs diachroniques dans la catégorie " connotation " (qui perdrait en même temps un peu de son flou), voire partiellement - pour autant que la fonction intra-musicale est concernée - dans la " dénotation ". 


\section{NOTES}

1. Voir les remarques de Serge Gut au sujet de "l'illogisme d'expliquer l'histoire de la musique à partir des 'grands compositeurs' " dans son compte rendu sur Dahlhaus (1983).

2. Ce point de vue est développé, par exemple, dans Fónagy (1956).

3. Voir par exemple la situation de la préposition allemande wegen, traditionnellement suivie du génitif ; celui-ci est de plus en plus concurrencé par le datif, surtout en style familier.

4. Ainsi, le système nominal français a progressé bien plus que le système verbal sur la voie de la "simplification" qui revient au remplacement des formes multi-fonctionnelles - c'est-à-dire complexes, avec des morphèmes agglutinés (désinences, pré- et suffixes) - par des formes analytiques aux unités mono-fonctionnelles (préposition + article + nom pour exprimer le « cas », etc.).

5. Voir les remarques et critiques publiées par Gauthier-Villars sous les pseudonymes « Willy » et " L'ouvreuse du cirque d'été " entre 1890 et 1901.

6. Comme le Poème d'avril (1866) ou les Expressions lyriques (publ. en 1913).

7. Pour cet aspect, voir Marschall (1983).

8. Ce qui est à ne pas confondre avec le statut de " compositeur de premier rang " évoqué plus haut. On peut faire école en restant au second rang ; cela semble plutôt être la règle. Les très grands restent inégalés ou sont misérablement imités.

9. Explication des indications numériques : le nom de l'oeuvre est suivi d'un numéro en chiffres romains indiquant l'acte, éventuellement accompagné d'un petit chiffre placé en haut et à droite indiquant le tableau, le tout étant suivi d'un numéro indiquant la mesure à l'intérieur de l'acte qui correspond à la première mesure de l'exemple.

10. Pour reprendre un terme d'André Schaeffner.

11. Manfred Kelkel étudie cet aspect dans sa thèse d'État sur l'opéra naturaliste (Paris-Sorbonne 1982, pas encore publiée).

12. Ce qui n'infirme pas le fait que certaines formes et structures de la musique instrumentale sont, historiquement parlant, calquées sur des modèles vocaux (telle la cadence de concerto sur le chant en vocalises, des notions comme cantabile sur certaines qualités du chant humain etc.)

13. La filiation Wagner-Debussy étant souvent soulignée (voir l'article de Hirsbrunner 1983), il convient d'objecter que, si le melos wagnérien est aussi ancré dans la mise en musique du verbe, il est en revanche extrèmement " tendu ", voir souvent compact. Cela nous semble résulter a) de son intégration plus ou moins parfaite au corps orchestral (ce qui n'est pas la même chose qu'une mélodie instrumentale conçue d'après des critères vocaux), b) de la forte stylisation et de l'allongement des durées, c) du fait de concevoir la mélodie, essentiellement, comme résultante de la progression harmonique (voir la partie 5 de notre article). 
88

14. Considérer l'importance du rythme $\iint^{3}$ chez Bruckner.

15. Il s'agit du $20^{\mathrm{e}}$ motet selon la numérotation dans l'édition complète de F. Ludwig.

16. Pour un schéma $A-A-B$ agissant au niveau de la période et du thème entier, voir la musique de la salle des joueurs dans Manon, IV, (mm. 85-89).

17. Elle a sans doute des origines encore plus profondes. En outre, la littérature italienne connaît plusieurs formes dérivant de la structure A-A-B.

18. Pour toute cette problématique, voir Ruwet (1972) : 111 et 135136 (au sujet de la répétition comme critère d'analyse) ainsi que 71-73 et 90-99 (au sujet du rôle de la répétition chez Debussy). On se reportera en particulier au début de Nuages (mm. 1 à 4) de Debussy, cité par Ruwet (1972: 73).

19. Au-delà de quatre sons, la définition reste possible mais devient trop complexe et voulue, les formations étant réellement perçues comme a-tonales.

20. L'instrumentation massenétiste n'a jamais fait l'objet, jusqu'à l'heure actuelle, d'une étude détaillée. 


\section{RÉFÉRENCES}

FÓNAGY, I.

1956: "Über den Verlauf des Lautwandels " , Acta Linguistica Hungarica. Budapest.

GRÉTRY, A.M.

1789: Mémoires ou Essai sur la musique. Paris. GUT, S.

1983: Compte-rendu de Dalhaus, C., "Die Musik des 19. Jahrhunderts", Revue de musicologie, 69/1, 120-27.

HARDING, J.

1970: Massenet. Londres : Dent.

HIRSBRUNNER, T.

1983: "Wagnérisme et wagnériens ", Lendemains, VIII/31-32, 88-93.

MARSCHALL, G.

1979: Massenet et la fixation de la forme mélodique française : Thèse de doctorat de $3^{\mathrm{e}}$ cycle. Paris : Sorbonne et Bibliothèque Nationale (exemplaires dactylographiés).

MARSCHALL, G.

1983: "Adaptation von Weltliteratur im drame lyrique ", Lendemains, VIII/31-32, 70-79.

RUWET, N.

1972: Langage, Musique, Poésie. Paris : Le Seuil.

SCHNEIDER, L.

1908: Massenet, l'homme et le musicien. Paris: Carteret.

1926: Massenet. Paris : Fasquelle (Reprint). 